\title{
BALANCED GOALCARDS Combining Goal Analysis and Balanced Scorecards
}

\author{
Alberto Siena \\ Center for Scientific and Technological Research FBK-Irst, via Sommarive 18, Trento, Italy \\ siena@fbk.eu \\ Alessio Bonetti, Paolo Giorgini \\ University of Trento, via Sommarive 14, Trento, Italy \\ alessio.bonetti@unitn.it,paolo.giorgini@unitn.it
}

Keywords: Software Engineering, Requirements Engineering, Business Strategy, Goal Analysis.

\begin{abstract}
Today's Information Systems are complex systems that have to deal with a variety of different and potentially conflicting needs. Capturing their strategic requirements is a critical activity, as it must answer at the same time to software and corporate goals. In this paper, we introduce the economic foundations of strategic requirements. We propose a novel conceptual framework for requirements modeling and validation, based on economic and business strategy theory. The soundness of the framework is also evaluated, by presenting the result of its application to a real case study.
\end{abstract}

\section{Introduction}

Understanding and analysing the purpose of a software system before defining its desired functionalities, results crucial and more and more mandatory in the development of modern information systems (Fuxman et al., 2001). Early requirements analysis (Giorgini et al., 2003; Alencar et al., 2000) is currently gaining popularity in the requirements engineering community as an effective way to understand the reasons of a new system with respect to the organisational setting in which it will operate. In this context, goal-oriented techniques have been proposed in the last decade (Rolland, 2003; Giorgini et al., 2005; Delor et al., 2003) to answer why questions, besides what and how, regarding system functionality. Answers to why questions ultimately link system functionality to stakeholder needs, preferences and objectives.

Goal analysis techniques (Dardenne et al., 1993) are useful to understand the structure and the correlations among goals, their decomposition into more fine-grained sub-goals, and their relation with operational plans. Moreover, reasoning techniques applied to goal models (Giorgini et al., 2002) can be very useful to verify properties of the model and possibly to support the analyst in the conflict resolution process. Although these techniques result very useful to rea- son about single goal models, they are inadequate to support strategic decisions of an organisation. This is mainly due to the fact that they assume the perspective of the designer of the system and do not consider other dimensions like the business or financial needs that are crucial in decision process of an organisation.

In this paper, we revise the Tropos methodology (Bresciani et al., 2004) extending its goal reasoning framework (Giorgini et al., 2005) with a more enterprise and business oriented approach, the balanced scorecards. The adoption of balanced scorecards allows the analyst to have a more comprehensive vision of the enterprise and consequently to adopt solutions that can be related to its strategic decisional process. The purpose of this paper is twofold. On the one hand, it introduces the conceptual framework and the methodology for guiding the analyst in the requirements analysis process, by assuming and combing multiple perspectives of analysis (i.e., financial perspective, internal processes perspective, customer perspective, learning and growth perspective). On the other hand, it evaluates such a framework with respect to its actual contribution to the software engineering process, by means of a real world example.

The paper is structured as follows. Section 2 presents our experience with a requirements analysis case and the problems it rises. Section 3 introduces the underlying concepts of goal analysis techniques 
and the Balanced Scorecards business modelling approach. Section 4 presents the new Balanced Goalcards framework. Section 5 discusses the results that emerge from the application of the framework to our case. Section 6 concludes the paper.

\section{An IS for logistics}

Frioli S.n.C. is a small Italian transport company located in the Trentino region, interested in developing a new information system to support its business. The company was founded in the sixties as a family company, and today it has five associates and four full-time employees. It owns five trucks and four trailers, and it transports both construction material (mainly cement) and undifferentiated goods. The transport activity is $80 \%$ in northern Italy, $10 \%$ in the rest of Italy, and $10 \%$ among Austria, Germany and France. Its costs are mainly related to fuel, insurance and road tolls. First need of the company is to optimise travels, and a new information system seems to be a necessary step in this direction. Our purpose is to gather the requirements for such an information system, ensuring them to be aligned with the actual business culture of the company.

Requirements have been initially collected after a number of meetings with people in the company. The main objective of these meetings was to understand the company's strategy and related activities. Unfortunately, after that it was not possible to have further meetings with the company (mainly for reasonable business constraints). This methodological constraint is the major motivation for our work. In a perfect world, with full information and unlimited resources, we could elicitate the requirements perfectly with effectiveness and efficiency. But in the real world, the challenge of software development is to overcome any kind of limitations - time constraints, budget constraints, communication obstacles, domain-specific skills, implicit information, and so on - to deliver the right solution at the right time and for the right market (Ebert, 2005).

Our experience with the company has confirmed such a problem. A modelling session followed the interviews, with the purpose to model and formally analyse the models. Already during the modelling phase, we have encountered suspicious inconsistencies. For instance, managers declared interest in reducing costs but they were not interested to reduce phone customer support and off-line marketing techniques in favour of on-line services. The reason of this can be shortly explained as follows: the company has currently a positioning in the market and in the local community and doesn't want to lose it. Its positioning is built on top of the company's philosophy that is implicit and depends on many factors, such as quality of directors and employees, local market characteristics, history and structure of the company, results obtained, etc. In this scenario, on-line marketing can produce a very negative impact and change heavily the customers' perception of the company. So, even if the company wants to reduce costs, choosing the lower-cost solution is not the right solution.

These and other considerations showed clearly the need to:

i) support the analyst in capturing during the interviews both technical requirements and business strategy needs;

ii) provide a formalism to represent business entities in the software development process.

\section{Background}

\subsection{Tropos and Goal Analysis}

Tropos (Bresciani et al., 2004) is an agent-oriented software development methodology, tailored to describe the system-to be in terms of its requirements. It is intended to capture both functional and nonfunctional requirements, and, as such, a special role is given to the capability to model and understand the stakeholders' goals. The methodology analyses the requirements of the system-to-be in terms of goal models. Goals basically represent the functional requirements, while the softgoals represent the nonfunctional requirements of the system.

Goal models are represented by means of goal graphs composed of goal nodes and goal relations. Goal relations can be AND and OR decomposition relations; or, they can be contribution relations, partial - the "+" and "-" relations - and full - "++" and “-_" relations. In practice, a goal graph can be seen as a set of and/or trees whose nodes are connected by contribution relations arcs. Root goals are roots of and/or trees, whilst leaf goals are either leaves or nodes which are not part of the trees.

For each goal of a goal graph, we consider three values representing the current evidence of satisfiability and deniability of goal: $\mathrm{F}$ (full), $\mathrm{P}$ (partial), $\mathrm{N}$ (none). We admit also conflicting situations in which we have both evidence for satisfaction and denial of a goal. So for instance, we may have that for goal $\mathrm{G}$ we have fully $(\mathrm{F})$ evidence for the satisfaction and at the same time partial $(\mathrm{P})$ evidence for denial. Such an evidence is either known a priori or is the desired 
one. In both cases, the conflicts arise by reasoning on the graphs with the techniques explained below.

On goal graphs, it is possible to analyse it with both forward reasoning and backward reasoning.

Forward Reasoning. Given an initial values assignment to some goals, input goals from now on (typically leaf goals), forward reasoning focuses on the forward propagation of these initial values to all other goals of the graph according to the rules described in (Giorgini et al., 2005). Initial values represent the evidence available about the satisfaction and the denial of a specific goal, namely evidence about the state of the goal. After the forward propagation of the initial values, the user can look the final values of the goals of interest, target goals from now on (typically root goals), and reveal possible conflicts. In other words, the user observes the effects of the initial values over the goals of interests.

Backward Reasoning Backward reasoning focuses on the backward search of the possible input values leading to some desired final value, under desired constraints. We set the desired final values of the target goals, and we want to find possible initial assignments to the input goals which would cause the desired final values of the target goals by forward propagation. We may also add some desired constraints, and decide to avoid strong/medium/weak conflicts.

The present work is based on the consideration that this kind of systematic analysis of the stakeholders' goals is necessarily general-purpose and performed from the perspective of the requirements engineer. As such, it provides little help in understanding the business specific requirements of the organisation. To overcome these difficulties, we refer to the "Balanced Scorecard" approach.

\subsection{Balanced Scorecards}

The Balanced Scorecards (BSC) framework was introduced by Kaplan and Norton in the early nineties (Norton and Kaplan, 1992) as a new strategy management approach, able to overcome the limitations they found in the then existing management methodologies. Essentially, pre-existing financial analysis techniques used to focus on monetary indicators, without taking into account non-measurable capitals of a company, such as knowledge or customers loyalty. As opposite, the BSC approach relies on three basic ideas (Kaplan and Norton, 2001): i) both the material and immaterial objectives are important for the company; ii) the objectives, material and immaterial, can be numerically measured via properly chosen metrics; iii) the strategy is the resultant of the balancing of different kinds of metrics. In a broad sense, the strategy consists in the set goals, which will determine the success of the organisation in the market (Porter, 1996). The strategy is the actual realisation of the mission, values and vision: the Mission statement describes the reason of being of the organisation and its overall purpose; the Business Values represent the organisation's culture, and turn out in the priorities that drive the organisation's choices; the Vision describes the goals to be pursued in the medium and long term, and how the organisation wants to be perceived from the market.

In the BSC approach, the strategy is bounded with a conceptual tool, the Strategic Map (Kaplan and Norton, 1996). It is commonly represented as a diagram, containing goals and their inter-connections. The connections are cause-effect relationships, meaning that the achievement of a goal brings to the achievement of its consequent. The strategic map is comprised by four different perspectives, i.e., the the financial perspective, the internal processes perspective, the customer perspective and the growth perspective.

The Economic-Financial Perspective. This perspective looks at the company from the point of view of the profitability, as well as solvency, liquidity, stability and so on. It expresses how well are the companys finances managed to achieve the mission. The metrics associated with this perspective are monetary and economic such as ROI, ROE, and more low-level values.

The Customer Perspective. Customers are the key stakeholders for the success of a company, so it is important to identify the target customer and define the properties that meet his needs. The ultimate purpose is to make products attractive for the customers.

The Internal Processes Perspective. The goals associated with this perspective are those that can have an impact on the internal effectiveness and efficiency of the company. Also, the goals that attain human and organisational resources are relevant, and can be measured by metrics such as are time, turnaround time, internal communications rating and so on. For instance, the choice to use e-mails instead of the telephone could improve the processes.

The Learning and Growth Perspective. The fourth perspective describes the long-term success factors. It is important to understand the characteristics that should equip the human, informative and organisational resources. So for instance, the choice to train the employees to use IT resources could allow the company to stay on the market also in the evolving global economy.

The representation capabilities of a strategic map may appear indeed limited. For instance, cause- 
effect relationships are too restrictive and do not allow the analyst to represent all the possible relationships among goals. Also, it is not possible to express negative effects; it is not possible to specify partial contributions among goals; and it is not possible to express conflicts. Finally, relationships among goals are not enough fine-grained: when multiple causeeffect implications exist, it is unclear what can happen if some goals cannot be satisfied. However, besides these limitations the conceptual expressiveness of the BSC approach seems promising, since it can capture a business-centred entity, such as the strategy, in quite a formal way. Thus, we aim at extending the representation capabilities of Tropos goal diagrams with the conceptual expressiveness of the BSC.

\section{Balanced Goalcards}

With the term "Balanced Goalcards" we refer to a novel approach for conceptual modelling that aims at aligning the business-centred needs of an organisation with IT-oriented requirements. The approach extends the $i * /$ Tropos methodology in both its modelling and analysis capabilities, and turns out in the ability to capture strategic requirements that are consistent with economic principles. The methodological framework of the approach is shown in Fig. 1. Shortly, the first step is the domain modelling and consists in the definition of the basic organisational settings. The actors are identified and their strategic dependencies are modelled. This phase follows exactly the Tropos methodology guidelines (see (Bresciani et al., 2004) for more details). Then, in the strategy modelling, the business context is modelled. Firstly, the mission of the organisation should be made clear, and then business values and vision. Using as leading parameters these root entities, the four different perspectives are modelled separately. The perspectives are then joint in a goal diagram that represents the Strategic Map. Finally, the map is validated along three dimensions: conflicts detection and resolution, minimisation of costs, and evaluation of unpredictable events. The result of the validation is the actual requirements system.

\subsection{Strategy modelling}

The idea is that the Tropos early requirements phase is led by the emergence of the strategic map. The strategic map is represented as a Tropos goal diagram, so that BSC's cause-effect relations are replaced by Tropos relations. AND-decompositions are used, when multiple cause-effect relations exist, and there is the

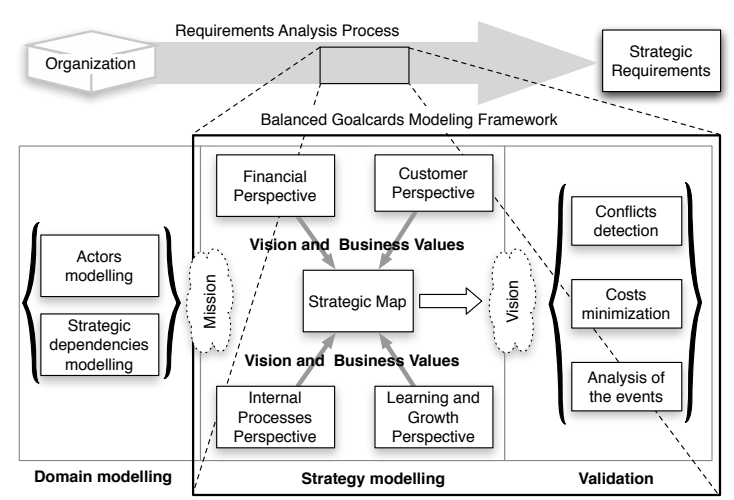

Fig. 1: The Balanced Goalcards methodological framework

evidence that the decomposed goal can't be reached if at least one of the sub-goals is not achieved. ORdecompositions are used, when multiple cause-effect relations exist, but achieving one of the sub-goal is enough to consider achieved the parent goal. If there is no clear evidence of a decomposition, a contribution link is used. Carefully evaluating the contribution metrics ("+", “-”, “++" and "-_,", see section 3 for the guidelines) allows the designer to describe in a more precise and realistic way the mutual influence among goals.

Mission. The concept of mission is mapped as the root goal. Frioli is a transportation company, so we have a root in the goal "Delivery service be fulfilled" as in Fig. 2(a). The root goal is further analysed by a decomposition into more operational goals ("Orders be received", "Goods be delivered" and so on). There could also be more that one root, if the company's business is wider.

Business Values. The business values are represented as softgoals. They emerge from both, an explicit indication of the organisation to be modelled, and the perception of the analyst. For instance, "Customer loyalty", "Timeliness", "Care corporate image", and so on (Fig. 2(b)) are business values. They are linked in weak cause-effect relations (represented as contributions in the picture). The "Long-term value for the associates" general-purpose goal is possibly reached directly, via a "Growth" of the company; or indirectly, via the "Customers loyalty" given by the "Quality of service". "Effectiveness" is related in particular with the inner processes of the company, whereas "Care corporate image" refers to how to company is perceived by the customer.

Vision. The vision represents the link between what the organisation is and what it wants to be. For the Frioli company, no vision has been clearly identified. This mainly because it is implicit in the managers' ra- 


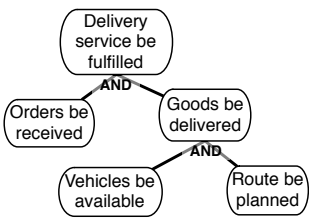

(a)

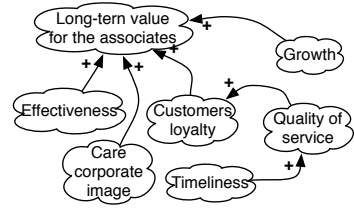

(b)
Fig. 2: (a) The mission of the company. (b) The business values. Ovals represent goals, and clouds represent softgoals.

tionales, and the challenge is to capture it and make it explicit, so that we can elicit consistent requirements. Strategy. We want to make the strategy to emerge during the goal modelling, through the building and evaluation of the strategic map. We want to capture the business needs, and this means we need also to model the business profile of the company. In order to do this, we adopt here the classical BSC-based modelling approach. In detail, we build our goal model by taking into account the four perspective mentioned above: the economic-financial perspective, the customer perspective, the internal processes perspective and the learning and growth perspective.

Economic-financial perspective. From the economic perspective, we observe an important effort of the Frioli company to contain costs (see Fig. 3). There are two kinds of costs: management costs and supply costs. Supply costs are intrinsic to the transport activity, such as fuel and tolls. On the other hand, management costs are related to the administration of the business; TLC are an important part, but also the extra payments the arise from unforeseens.

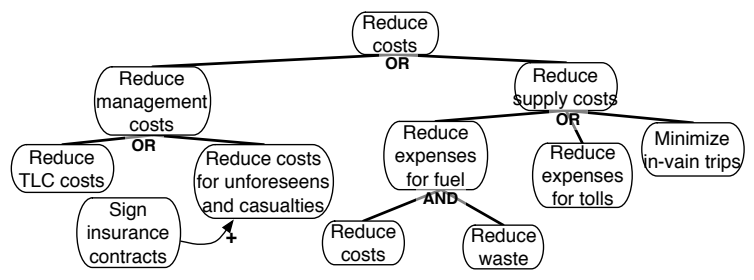

Fig. 3: The Economic-financial Perspective.

Customer perspective. From this perspective, it is important to understand in which way a company can be attractive for the customer (Fig. 4). The overall image of the company ("Care corporate image") is important, as well as the details, such as the look of the personnel and of the documents. The communications with the customer ("Care the communications with customers") are important for the customers to be loyal to the company. Also, an important goal is to be able to offer an international transportation service.
Even if not frequent, the lack of this service could affect the perception of the customer in the company's capabilities.

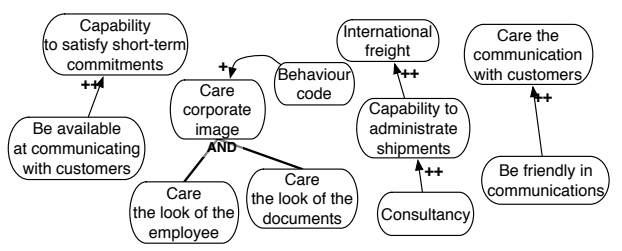

Fig. 4: The Customer Perspective.

Internal processes perspective. Economic-financial goals and customer's strategy have to be translated into internal processes. Notice that we don't want to actually model the sequences of activities that form the company's business processes. What we want to capture here is the why of the processes. The processes shall allow the company to achieve its goals, so we model only the low-level goals that the internal activities are expected to fulfil (Fig. 5).

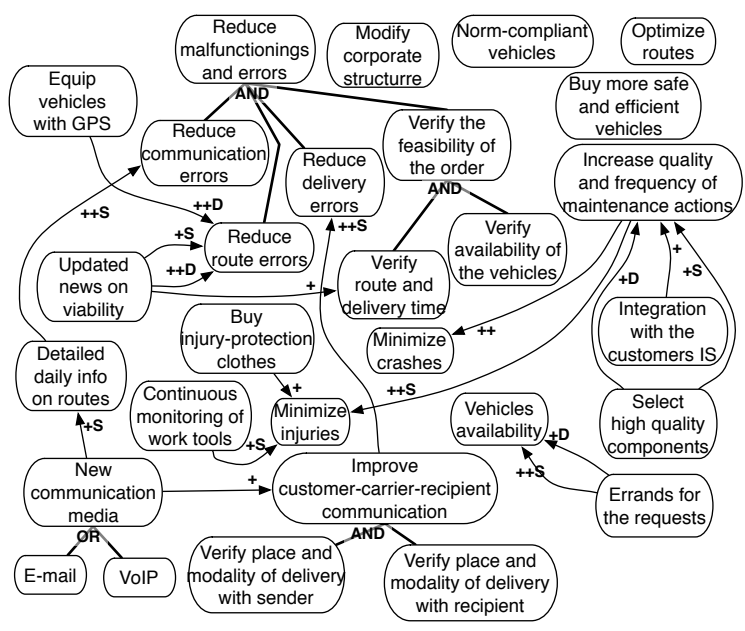

Fig. 5: The Internal Processes Perspective.

Learning and growth perspective. From this perspective, the company has a little margin of technical improvement. For instance, it could acquire new tools (for self-made reparations) or train the personnel (Fig. 6). Some other goals are related to the acquisition of new customers. More ambitious growth plans, such as investments in new market segments, are not present in the company.

\subsection{Validation of the Strategic Map}

The last step consists in putting together the perspectives and balance them into a consistent strategy. One 


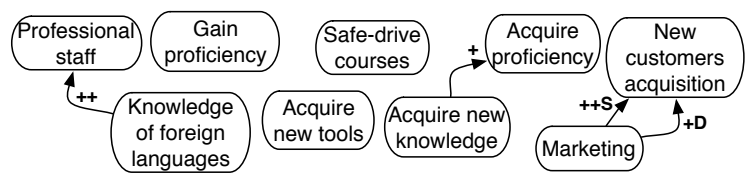

Fig. 6: The Learning and Growth Perspective.

of the strength points of the original BSC framework is its simplicity; using a goal diagram based approach causes the level of complexity to grow up (see Fig. 7), and this raises the need for formal analysis able verify and validate the models. The first problem (verification) can be solved with goal analysis techniques. The second problem (validation) is more complex, since it requires to align the models with economic principles. For this purpose, we have defined an analysis methodology comprised by three steps: conflicts resolution, costs minimisation and risk prevention.

Conflicts resolution. A conflict is a situation where the satisfaction of some subgoals prevents (fully or partially) the top goals from being fulfilled. Such kind of situation is extremely dangerous, since an unseen conflict can undermine the enforcement of the strategy. But it results difficult to be detected, specially when the strategic map becomes complex. The procedure that we use for conflicts detection and resolution is the following: $i$ ) we execute backward reasoning in order to find a possible vales assignment for leaf nodes that satisfy all the top goals; ii) if the solution introduces a conflict for some of the top goal we start again backward reasoning for each of the conflicting goal; iii) the whole goal model is then modified accordingly to the partial results obtained in the previous step.

So for example, in Fig. 7, the goal "Verify the feasibility of the order", which was introduced in order to "Reduce malfunctioning and errors", caused a conflict with the goal "Reduce management costs". Since the company privileges the financial perspective over the internal processes one, the first goal has been discarded.

Costs minimisation. Due to OR-decompositions and multiple contribution relations, different strategies can be adopted; i.e., it is possible to find different values assignments to the leaf goals that satisfy the top goals without any critical conflict. To select one of them we recall the BSC fundamentals, arguing that each alternative has a different cost for the organisation. So we assign to each goal a numerical value, which represent its cost. If possible, we evaluate its monetary cost; so for instance the actual cost of the "Consultancy" can be accurately estimated. Otherwise, we search for a suitable metrics; for instance, for transport companies "timeliness" can be evaluated and translated into a numerical value. Costs are then associated to goals as meta tags, so that each possible strategy will have by this way a corresponding weigh in term of its resulting cost. The selection of the best strategy will be based on the comparison of that costs. For instance, in Fig. 7, the goal of obtaining "Customers loyalty" can be achieved either adopting a marketing strategy (e.g., promotional campaigns) or introducing a dedicated software able to reduce delivery errors. However, a new software can be very expensive and adopting marketing-based strategy could be more convenient.

Risk prevention. Risk is something that heavily affects a company's life. Risky events are outside the control of an organisation and can prevent its strategy to be accomplished. We take into account this problem by introducing in the diagrams a new entity - the "Event". Events are linked via $-{ }_{S}$ contribution relations to one or more goals. So, if an event occurs (i.e., its SAT value equals to $\mathrm{P}$ or $\mathrm{F}$ ), then the affected goal is inhibited. We have no control over the occurrence of the event; however, by assigning the SAT value to the event, we can perform bottom-up analysis and see what is the potential effect of the event.

For example, in Fig. 7 the event "Crashes" can potentially compromise the whole long-term strategy having a negative impact on the reduction of costs.

\section{Evaluation}

In order to verify our approach, we have implemented a CASE tool, the B-GRTool (BalancedGoalCards Tool). The tool has been implemented as an extension of the GR-Tool (Giorgini et al., 2005) and maintains all its functionalities, including reasoning techniques like forward and backward reasoning. Besides the standard GR-Tool scenarios, the BGRTool supports views on single scenarios that are used to build the balanced perspectives. We used the tool to model the strategy for Frioli S.n.C. according to the approach shown in previous section. An almost complete goal model is illustrated in Fig. 7. It contains the mission and values of the company, together with the four perspectives; notice that during the validation phase, we have completed and refined the model by establishing further relations (contributions and decompositions). Table 1 shows the metrics that result from the goalcards; due to lack of space, only a subset of the goals can be shown. The four scenarios correspond to alternative strategies, each of which gives different priorities to different goals.

Scenario 1. The first scenario shows the current strat- 


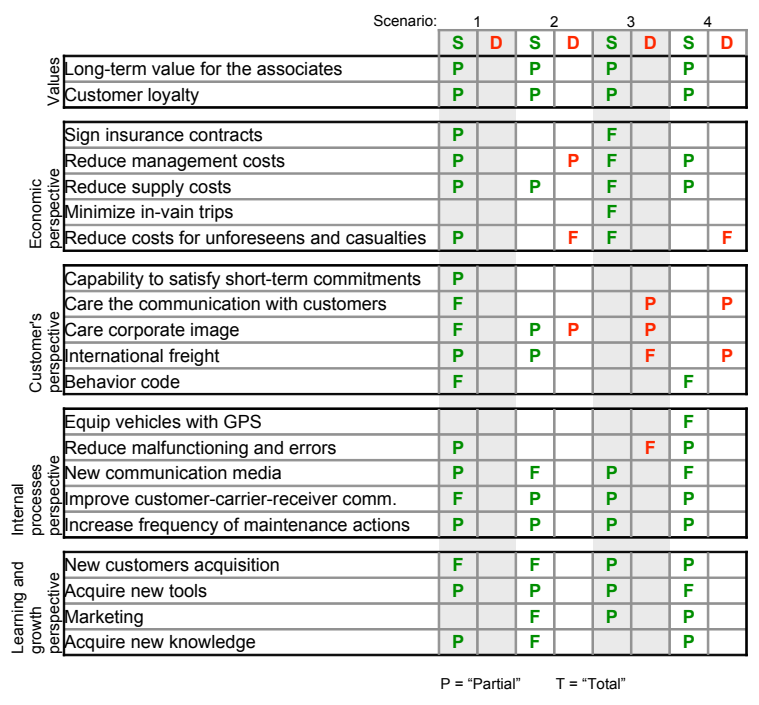

Table 1: Perspective-based comparison of four possible scenarios. The "S" columns contain Satisfiability values, whereas the "D" columns contain Deniability values.

egy of the company and reflects its business values. In this case, some goals result more important than others. For instance, it is extremely important the "Customer loyalty" obtained offering a reliable service. This requires a particular attention to "Care corporate image" and to "Care the communication with customers".

Scenario 2. The second scenario proposes a strategy for a growth-oriented company. The focus of the analysis is on goals such as "Acquire new tools" or "New customers acquisition". What we obtain is a strategy that privileges the growth, but goals such as "Reduce management costs" and "Reduce costs for unforeseens and casualties" are denied. If a company wants to grow, it is extremely difficult to reduce at the same time the costs.

Scenario 3. The third scenario describes a strategy for a company that wants to reduce costs. In this case, the focus is on the economic and the internal processes perspective, and particularly on goals such as "Reduce management costs", "Reduce maintenance costs", and "Reduce expenses for fuel". The resulting strategy allows the company to satisfy all goals, but suggests to abandon the international freight. Also, it is interesting to see that in this strategy some non essential goals such as "Care the communication with customers" and "Care corporate image" are denied.

Scenario 4. The last scenario proposes to reach the top goals by investing in innovation. In this case the learning and growth perspective has again a relevant influence on the strategy, but caring at the same time the internal processes one. The resulting strategy is

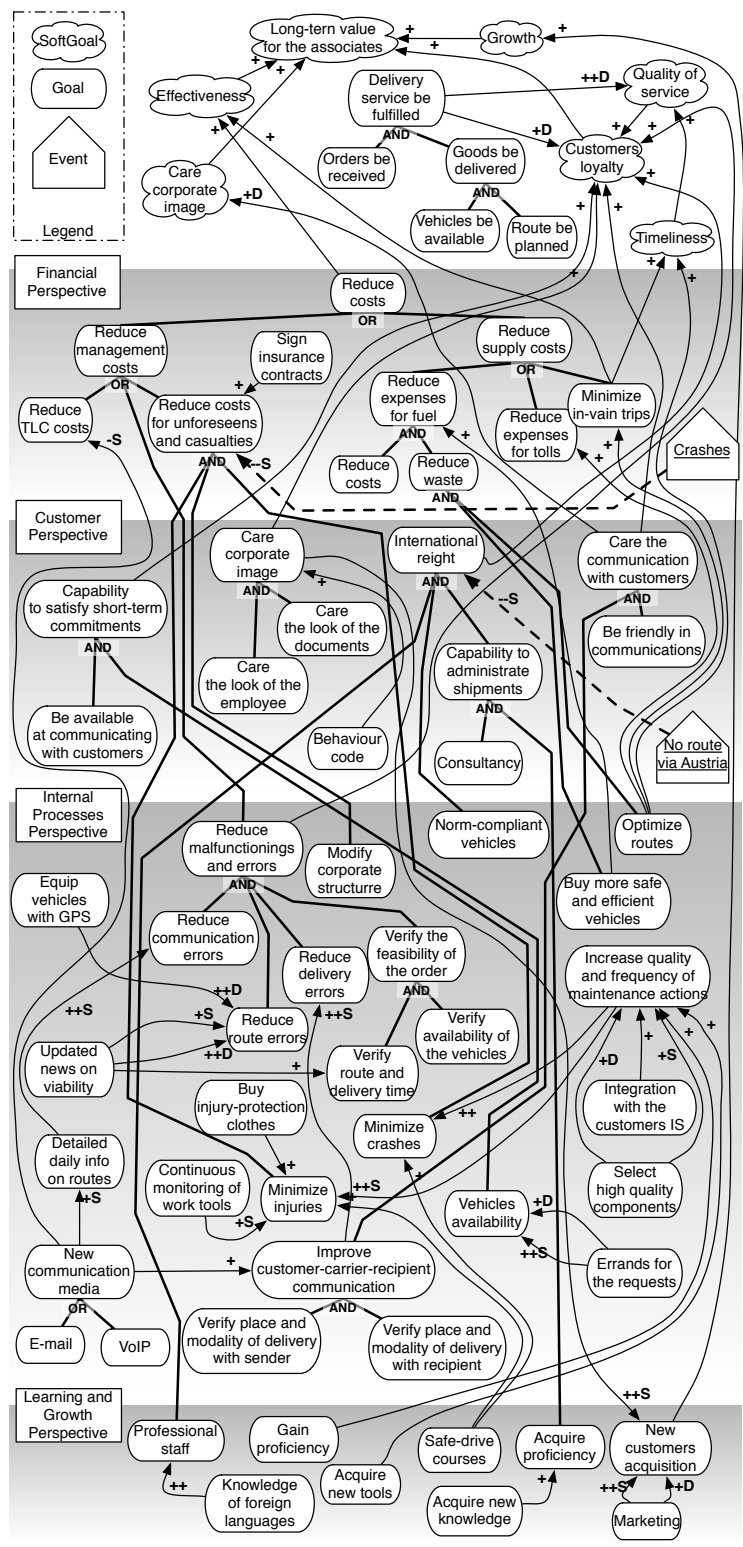

Fig. 7: An (almost) complete goal model for the Frioli S.n.C. case study

similar to the one of scenario 2 , but it is now possible to contain costs.

Some interesting results come from the case study. Despite their claim of the "Growth" as a business value, the actual strategy does not reflect such a will. It is possible to see in Fig. 7 that the learning and growth perspective has a few number of goals. The scenarios above also confirm this perception. So we observe that, despite the fact that the company wants to grow, its implicit values do actually privilege stability. This observation is reinforced by a look at the customer perspective: it has an important impact on 
the realisation of the business values. In particular, we see that many goals exist in order to satisfy the "Customer loyalty" soft-goal. Through the customer loyalty, the general-purpose "Long-term value for the associates" is intended to be reached. Thus, the company seems to have a well-established relation with customers, and wants to keep it, without going further into market risks. So the resulting requirements system should privilege this status quo arrangement.

\section{Conclusions}

The importance of business criteria is explicitly recognised in particular in the e-Commerce engineering, where value exchanges plays a role in understanding and generating requirements for the system (Gordijn and Akkermans, 2003). Also, in eBusiness, some approaches exist, which focus on the need for the alignment of IT and business strategy (Bleistein et al., 2004; Grembergen and Saull, 2001). In this paper we have presented a new methodological framework for modelling requirements and validating them against a business strategy: goal graphs are used to represent the strategic map, and the economic metrics are associated to goals' satisfiability and deniability; this allows to reason on the metrics and analyse the diagrams, building balanced requirements systems. We have reported the use of the framework in our experience with a transport company, trying to evaluate the results by comparing different scenarios and estimating the effectiveness gained in gathering requirements.

\section{REFERENCES}

Alencar, F., Castro, J., Cysneiros, L., and Mylopoulos, J. (2000). From early requirements modeled by the $\mathrm{i}^{*}$ technique to later requirements modeled in precise UML. In Anais do III Workshop em Engenharia de Requisitos, pages pp. 92-109, Rio de Janeiro, Brazil.

Bleistein, S. J., Aurum, A., Cox, K., and Ray, P. K. (2004). Strategy-oriented alignment in requirements engineering: Linking business strategy to requirements of e-business systems using the soare approach. volume 36.

Bresciani, P., Giorgini, P., Giunchiglia, F., Mylopoulos, J., and Perini, A. (2004). Tropos: An agent-oriented software development methodology. Journal of Autonomous Agents and Multi-Agent Systems, 8(8):203 $-236$.

Dardenne, A., van Lamsweerde, A., and Fickas, S. (1993). Goal-directed requirements acquisition. Science of Computer Programming, 20(1-2):3-50.
Delor, E., Darimont, R., and Rifaut, A. (2003). Software quality starts with the modelling of goal-oriented requirements. In 16th International Conference Software \& Systems Engineering and their Applications.

Ebert, C. (2005). Requirements before the requirements: Understanding the upstream impact. In $R E$ '05: Proceedings of the 13th IEEE International Conference on Requirements Engineering (RE'05), pages 117124, Washington, DC, USA. IEEE Computer Society.

Fuxman, A., Giorgini, P., Kolp, M., and Mylopoulos, J. (2001). Information systems as social structures. In Second International Conference on Formal Ontologies for Information Systems (FOIS-2001), Ogunquit, USA.

Giorgini, P., Kolp, M., and Mylopoulos, J. (2003). Organizational patterns for early requirements analysis. In the 15th Conference On Advanced Information Systems Engineering (CAiSE*03).

Giorgini, P., Mylopoulos, J., Nicchiarelli, E., and Sebastiani, R. (2002). Reasoning with goal models. In the 21st International Conference on conceptual Modeling (ER2002), Tampere, Finland. Springer Verlag.

Giorgini, P., Mylopoulos, J., and Sebastiani, R. (2005). Goal-oriented requirements analysis and reasoning in the tropos methodology. Engineering Applications of Artifcial Intelligence, 18/2.

Gordijn, J. and Akkermans, H. (2003). Value based requirements engineering: Exploring innovative e-commerce idea. Requirements Engineering Journal, 8(2):114 134.

Grembergen, W. and Saull, R. (2001). Aligning business and information technology through the balanced scorecard at a major canadian financial group: It's status measured with an it bsc maturity model. In HICSS '01: Proceedings of the 34th Annual Hawaii International Conference on System Sciences ( HICSS-34)Volume 8, page 8061, Washington, DC, USA. IEEE Computer Society.

Kaplan, R. and Norton, D. P. (1996). The Balanced Scorecard: Translating Strategy into Action. Massachusetts: Harvard Business School Press.

Kaplan, R. S. and Norton, D. P. (2001). The strategyfocused organization. how balanced scorecard companies thrive in the new business environment. Harvard Business School Press.

Niehaves, B. and Stirna, J. (2006). Participative enterprise modelling for balanced scorecard implementation. In Ljunberg J, A. M. e., editor, the Fourteenth European Conference on Information Systems, pages 286-298, Goteborg.

Norton, D. and Kaplan, R. (1992). The balanced scorecard: measures that drive performance. Harvard Business Review, 70 (1).

Porter, M. E. (1996). What is strategy? Harvard Business Review, 74(6):61-78.

Rolland, C. (2003). Reasoning with goals to engineer requirements. In 5th International Conference on Enterprise Information Systems, Angers, France, April 22-26. 